\title{
The implications of a high energy dense diet on the health of Ireland's children
}

\section{Laura Keyes}

\author{
School of Food and Nutritional Sciences, UCC
}

The energy density of foods is a major contributor to the global obesity epidemic - World Health Organisation 2003

\section{Figuring out dietary energy density}

Energy density (in relation to food) is a relatively simple concept; it refers to how densely packed with energy a food is and is calculated simply by dividing the calories in the food by the weight of the food. An apple is an example of a low energy dense food while a biscuit is a high energy dense food. Dietary Energy Density (DED), however, is slightly more complex; it is calculated by the same method but it is calculated for a person's diet rather than a single food. This is easier said than done because in a typical day people don't only eat food, they also drink beverages. Enter the biggest hurdle in calculating DED - drinks. The inclusion of drinks in the calculation of DED can be very misleading; a pint of water for example has zero calories and lots of weight, giving it an energy density of zero. Let's envisage a scenario where DED is calculated for two people, Paul and Fiona, including everything that they eat and drink for a day. Paul eats fruit for breakfast, a salad for lunch and a stir fry of vegetables for dinner and has one glass of orange juice; Fiona eats a chocolate bar for breakfast, no lunch and a 16 inch take-away pizza for dinner but drinks 10 pints of water. Fiona will end up with a lower DED than Paul, even though Fiona ate all the energy-dense foods. There is an on-going debate as to what is the best approach to deal with drinks and DED. The most obvious solution to this problem and the one that is gaining the most popularity in the scientific community is to remove all the beverages from the diet and calculate DED for the food portion of the diet only. When we apply this solution to the Paul and Fiona scenario, Paul will end up with a low DED and Fiona with a high DED, which better reflects their diets.

\section{Why look at dietary energy density}

High DED has been repeatedly associated with increased energy intake and weight gain. Studies have also shown that a high DED may increase a person's risk of having metabolic syndrome (a term used to describe a group of risk factors which when they occur together can increase the risk of coronary heart disease, stroke, or type II diabetes) and, worryingly, 
DED has been also linked in children with increased fatness. The World Health Organisation (WHO) has recognised energy density as an important dietary factor for determining obesity risk and a major contributor to the global obesity epidemic.

The crux of the link is that individuals can only fit a certain amount of food in their stomachs until they are full. One of the ways in which our body tells our brain that we are full is from the pressure sensors on the walls of our stomach; a full stomach puts pressure on sensors which begins a chemical reaction sending a message to the brain which ultimately leads to a signal to the hand to put the fork down! If an individual chooses a high energy dense diet; they can ingest a large amount of calories by eating a small amount of food, but they will still have room in their stomach and might still feel hungry and may eat again, whereas, an individual who selects a low energy dense diet is limited by the volume of their stomach, these people can eat more food for the same amount of calories becoming fuller faster.

That seems to make sense, so why would anyone eat a high energy dense diet? The simple answer is always the logical choice and in this case it's palatability - which is science for, yummy. High energy dense foods taste good. The creamy buttery taste of fat and the sweet deliciousness of sugar are the two most inherently desirable tastes to humans. Sweet foods like biscuits, because of their dryness are one of the most energy dense foods out there and fatty foods like 'chipper' chips are high energy dense because fat itself has so much energy per gram.

So now that we know how to calculate DED, why the nutritionists think it's important and how it works in the body, I'd like to point out another facet of DED - a high DED not only can affect our weight but can affect our nutrient intakes too, leading to poor dietary quality. This is an emerging concept and it is this aspect of DED that is the focus of my work. The following are results found in a nationally representative sample of Irish children; similar trends were found in teenagers and in adults, but as it is with our nation's children that we have the most opportunity to turn the tide, I thought I'd start there.

\section{The nutritional implications of a highly energy dense diet for Irish children}

In a nut shell, the higher the DED the less nutritious the diet becomes. To arrive at this statement I examined the effect of DED on the quality of children's diets using habitual food and drink consumption data of a representative sample of Irish children aged 512 years collected during the National Children's Food Survey (NCFS) (2003 - 2004). I calculated each child's DED and then, as there are no established cut-off points, split the children into three equal groups; those with low DED, medium DED and high DED.

The first result of note was that children with high DED ate less food but had the same amount of calories. The implications of this finding are that these children have less eating 
opportunities to consume the full cohort of nutrients that will allow for optimum nutrition. Protein, carbohydrate and fat are the building blocks of food, and the amount that they each contribute to the energy in a person's diet can have repercussions on the individual's health. As a general rule a higher quality diet will include more protein and carbohydrates and less fat. When compared to those with low DED, children with high DED had higher fat intakes and lower protein and carbohydrate intakes.

"Eat your brown bread, it's full of fibre" and "that's full of sugar, it'll rot your teeth" may just sound like something your mother used to say to make you eat the food you didn't like and stop you eating treats, but there is solid scientific fact behind them. Fibre is a key player in dietary quality and a great tool in staving off the obesity epidemic, and sugar, particularly added sugar, really will rot your teeth. Children with high DED had lower intakes of fibre and higher intakes of added sugar than their low DED counterparts.

Vitamins and minerals are crucial in a person's diet, in fact the word vitamins stems from the Latin for "vital for life". I examined 22 vitamins and minerals and found that the higher DED became the less of each and every vitamin and mineral the children consumed. There are certain targets set down for how much of each vitamin and mineral a person needs to remain healthy, a worrying outcome of my analysis is that the percentage of children that met these targets was far lower in the high DED group than in the low DED group.

It's all well and good to talk about protein, carbohydrates, fats, vitamins and minerals, but no one actually goes out and picks up a bar of iron or a slice of fat and eats it; they eat food. A very clear picture of the types of foods that children with high DED are eating emerged from my work. These kids were given by their parents or chose for themselves; real butter over a low fat spread, chips instead of potatoes and sugary drinks over water or juice. They had less fruit and veg in their diets and more chocolate and crisps than those with low DED. In essence they had more 'bad' food choices and ate more of these 'bad' foods than the low DED group.

\section{Conclusions}

The evidence that a high DED is associated with poorer dietary quality is quite compelling. Irish children with high DED eat more calories and fat and get fewer vitamins and minerals in their diets. Overall they have less nutritious diets and are at higher risk of being overweight and developing nutrition related diseases and illnesses in later life, than their low DED counterparts. We can improve the quality of their diets by promoting a low energy dense style diet; which includes lots of fruit and vegetables, potatoes, brown bread, yoghurts and fresh meat.

With thanks to the fieldworkers who collected the data, the Irish Government for funding the project under the National Development Plan 2000-2006 and Professor Albert Flynn for his supervision. 